\title{
Point-of-care ultrasonography: training, applications and barriers. A survey study
}

Gentle S Shrestha', Subhash P Acharya', Pramesh S Shrestha', Ninadini Shrestha', Hem R Paneru', Sharma Paudel', Benu Lohani', Saurabh Pradhan', Diptesh Aryal ${ }^{2}$, Navindra Bista', Bindu L Shah'3 , Amit S Bhattarai', Bashu D Parajuli', Megha Koirala', Pragya Acharya', Sabin Koirala ${ }^{4}$, Ashish G Amatya ${ }^{5}$, Sandip Bhandari ${ }^{5}$, Arjun Gurung ${ }^{6}$, Bibhush Shrestha ${ }^{6}$, Shailendra Sigdel ${ }^{6}$, Bigen M Shakya1, Niraj Keyal ${ }^{7}$, Anand Thakur ${ }^{8}$, Achyut Sharma ${ }^{6}$, Tamanna Bajracharya $^{9}$

'Tribhuvan University Teaching Hospital, Maharajgunj, Kathmandu, Nepal; ${ }^{2}$ Nepal Medici Hospital, Bhaisepati, Lalitpur, Nepal; ${ }^{3}$ National Academy of Medical Sciences, Mahaboudha, Kathmandu, Nepal; ${ }^{4} \mathrm{Hospital}$ for Advanced Medicine and Surgery, Dhumbarahi, Kathmandu, Nepal; ${ }^{5} \mathrm{Shahid}$ Gangalal National Heart Center, Bansbari, Kathmandu, Nepal; ${ }^{6}$ Manmohan Cardiothoracic Vascular and Transplant Center, Maharajgunj, Kathmandu, Nepal; ${ }^{7}$ B \& C Medical College and Teaching Hospital, Birtamod, Jhapa, Nepal; 8Grande International Hospital, Dhapasi, Kathmandu, Nepal; 9KIST Medical College and Teaching Hospital, Mahalaxmi Municipality, Lalitpur, Nepal.

Keywords: point-of-care ultrasonography, resource limited setting, survey

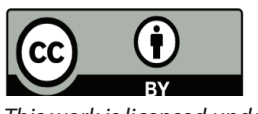

This work is licensed under a Creative Commons Attribution 4.0 Unported License.
Abstract

Background and Aims: Use of point-of-care ultrasound (POCUS) in acute care setting has rapidly increased and has potentials to guide patient management. This survey study aims to explore the usefulness of a one-day workshop and to elicit the perceived barriers for effective use of POCUS.

Methods: A total of 169 doctors who had attended one day Acute Care Ultrasound workshop were approached through email. Online link to access the survey created using Google forms was sent. The survey contained questions related to the details about the participants, feedback about the workshop, whether the workshop has helped to change the practice of the participants, availability of ultrasound machine during the daily practice and the perceived barriers for use of POCUS.

Results: A total of 41 responses were obtained. Majority of the participants had anaesthesiology as the base specialty followed by general practice. Most of them had ICU as their predominant working place, followed by emergency room and operating room. The workshop was found to be helpful by most of the participants. Majority of the participants ( 20 participants; 49\%) had ultrasound machine sometimes available during their daily practice. Only 20\% (8 participants) had ultrasound machine always available during their clinical practice. Similarly, 46\% (19 participants) considered lack of access to ultrasound machine as a barrier for application of POCUS. Significant number of participants considered lack of supervision and guidance (18 participants; $44 \%$ ) and lack of knowledge and skills (13 participants; $32 \%$ ) as the barriers.

Conclusions: Majority of the participants found the one-day workshop helpful. Doctors from various specialty, working in acute care setting had participated in the survey. Limited access to ultrasound machine, together with lack of adequate knowledge and skills were perceived as major barriers for effective use of point-of-care ultrasound.

Corresponding Author

Dr. Gentle S Shrestha, MD, FACC, EDIC, FCCP, FNCS

Associate Professor

Department of Anaesthesiology

Tribhuvan University Teaching Hospital

Maharajgunj, Kathmandu, Nepal

Tel No. 977-9841248584

Email: gentlesunder@hotmail.com 
and interpretation of sonography and the dissociation of the performing physician from the case is expected. ${ }^{2}$

Critical care ultrasonography (CCUS) can be categorized into general CCUS and echocardiography. The general CCUS encompasses thoracic (pleural and lung), abdominal and vascular ultrasonography. Critical care echocardiography is stratified into basic and advanced echocardiography. ${ }^{3}$ Acute care physicians should attain competence in CCUS. General critical care ultrasound and basic critical care echocardiography should be the integral part of the curriculum of critical care physicians. Critical care societies of own country should facilitate and encourage training in CCUS. ${ }^{4}$

In the recent decades, with the advent of compact, high quality and cheaper ultrasound units, the concept of POCUS is rapidly growing. 1 There is growing evidence in favor of POCUS. Use of transthoracic echocardiography during management of patient with sepsis may decrease mortality and can help to optimize use of fluids and vasopressors. ${ }^{5}$ The benefit of POCUS may be more pronounced in places with resource limitations like Nepal. However, barriers inherent to places with limited resources, like scarce access and availability of ultrasound machine and POCUS training, can be of significant concern. ${ }^{6}$ We performed an online survey among the participants of one day POCUS training to explore the impact of training, application of POCUS and perceived barriers.

\section{Materials and Methods}

A total of 169 doctors who had attended one day Acute Care Ultrasound workshop were approached through email. The one-day workshops consisted theoretical session in the first half followed by hands-on sessions. During theoretical sessions, principles of ultrasound, knobology, lung ultrasound vascular ultrasound (vascular access and diagnosis of deep vein thrombosis), focused assessment with sonography in trauma (FAST), basic echocardiography, neurological ultrasound (optic nerve sheath diameter), ultrasound during cardio-pulmonary resuscitation and ultrasound during shock were discussed. Handson sessions were conducted on the healthy volunteers with 6 to 8 participants in each station. Basic echocardiography, FAST scan, lung ultrasound, vascular ultrasound and ultrasound during shock were demonstrated and each participant scanned the healthy volunteers. Online link to access the survey created using Google forms, was sent on January 2019. The survey contained questions related to the details about the participants, feedback about the workshop, whether the workshop has helped to change the practice of the participants, availability of ultrasound machine during the daily practice and the perceived barriers for use of point-of-care ultrasonography. The respondents were kept anonymous. The responses were collected over next month. A total of 41 responses were obtained, which were analyzed.

\section{Results}

Age of the participants ranged from 24 to 42 years, with 29 (71\%) of them being males. Majority of the participants had anaesthesiology as the base specialty (17 participants; 53\%), followed by general practice (7 participants, $22 \%$ ), paediatrics, nephrology, endocrinology and critical care (2 participants each, $6 \%$ each). Most of the participants (19 participants; 46\%) spent majority of their time in the work place at operating room, followed by ICU (10 participants; $24 \%)$, emergency room (6 participants; $15 \%)$, outpatient department (5 participants; $12 \%$ ) and others (1 participant; 3\%) (Fig 1).

\section{Majority of time spent in the work place}

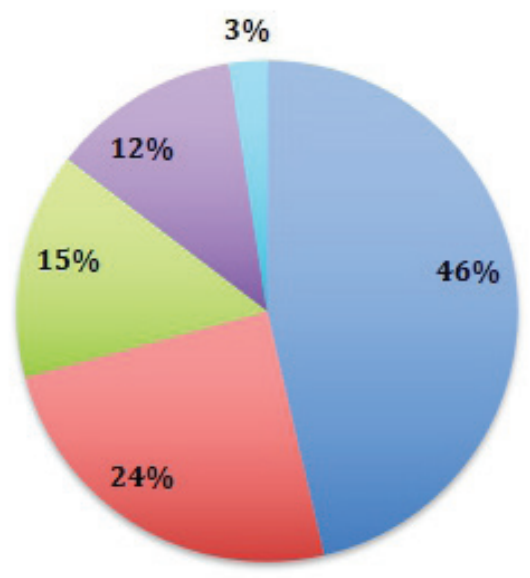

Fig 1. Majority of the time spent by the participants in the work place (1: Operating room; 2: ICU; 3: Emergency room; 4: Outpatient department; 5: Others)

About the contents of the workshop, 17 participants (42\%) considered the theoretical section to be very helpful, 21 participants (51\%) considered it helpful and 3 participants (7\%) as average. The hands-on session of the workshop was considered to be very helpful by 19 (46\%) of participants, to be helpful by another $19(46 \%)$ of participants and to be average by $3(8 \%)$ of participants. Of all the participants, 17 (42\%) found the workshop to be very helpful to change their daily practice, 16 (39\%) found it to be helpful, $7(17 \%)$ to be average and $1(2 \%)$ to be useless (Fig 2). Eleven (27\%) participants very often used ultrasound in their daily practice, $9(22 \%)$ used it often, $18(44 \%)$ used it sometimes, 2 (5\%) used it rarely and $1(2 \%)$ never used it (Fig 3). About the ease of access to ultrasound machine, $8(20 \%)$ had the machine always available at workplace, 10 (24\%) had it often available, 20 (49\%) had it sometimes available and $3(7 \%)$ had difficulty to access the machine (Fig 4).

\section{Usefulness of the workshop}

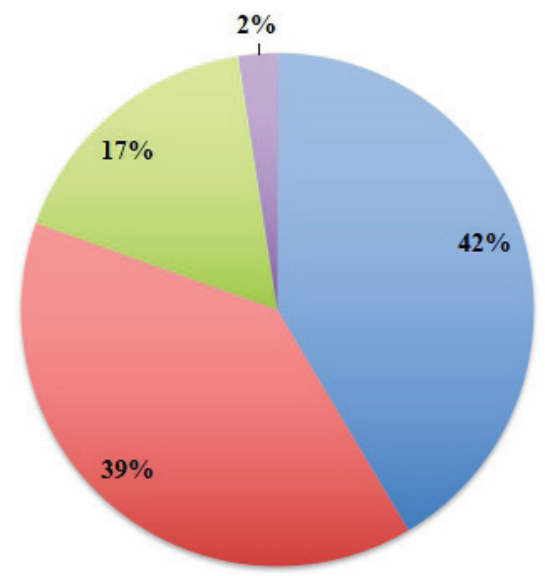

Fig 2. Usefulness of the workshop, as perceived by the participants (1: very helpful; 2: helpful; 3: average; 4 : useless) 


\section{Use of ultrasound in daily practice}

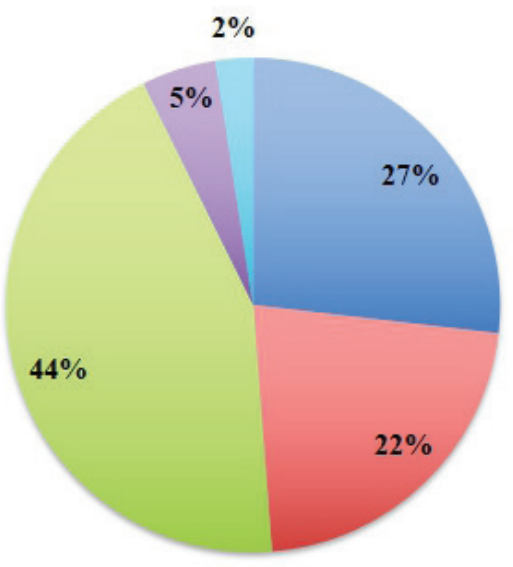

Fig 3. Use of ultrasound by the participants in their daily practice (1: very often; 2 : often; 3 : sometimes; 4 : rarely; 5 : never)

\section{Access to ultrasound machine}

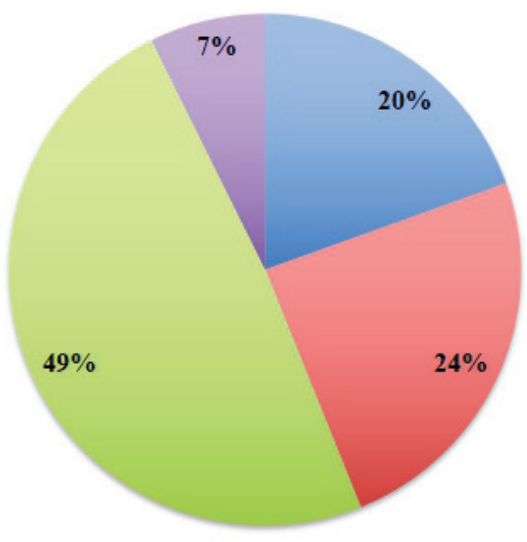

Fig 4. Ease of access to ultrasound machine at work place (1: always available; 2 : often available; 3 : sometimes available; 4 : difficult to access)

About the perceived barriers for application of point-of-care ultrasound, $19(46 \%)$ considered lack of access to ultrasound machine as the barrier, 18 (44\%) considered lack of supervision and guidance, 13 (32\%) considered lack of knowledge and skills and $12(29 \%)$ considered lack of time as the barriers.

\section{Discussion}

The use of POCUS in acute care setting is rising. ${ }^{1}$ There are multiple benefits of use of POCUS, including low cost, devoid of radiation hazards, available at bed side, repeatable and effectiveness in diagnosing multiple common pathologies in acute care settings. With these benefits, POCUS has the potential to improve diagnosis and management of multiple conditions, even in resource-limited settings. ${ }^{7,8}$

Majority of the participants had anaesthesiology as the base specialty followed by general practice. Most of them had ICU as their predominant working place, followed by emergency room and operating room. The workshop was found to be helpful by most of the participants. Majority of the participants (49\%) had ultrasound machine sometimes available during their daily practice. It correlates with the response of $44 \%$ of the participants who sometime use ultrasound. Only $20 \%$ of the participants had ultrasound machine always available during their clinical practice. Similarly, $46 \%$ of the participants considered lack of access to ultrasound machine as a barrier for application of POCUS. The findings are similar to those found in other developing countries. 6 Easy access and improved availability of ultrasound units can enhance the utility of POCUS in developing country like Nepal.

Significant number of participants considered lack of supervision and guidance (44\%) and lack of knowledge and skills (32\%) as the barriers to application of POCUS. Though the participants had attended a one-day Acute Care Ultrasound workshop, the knowledge and skills attained can likely be sub-optimal. We initiated the workshop on August 2013 and have trained over 200 doctors so far. The workshop aims to sensitize and educate the participants about the spectrum of use and potential benefits of POCUS, rather than assuring competency. The workshop is endorsed by Nepalese Society of Critical Care Medicine (NSCCM). Development of formal POCUS curriculum and incorporating it in the training program of the doctors who will be working in acute care settings can help to assure competency in various aspects of POCUS like image acquisition, interpretation and application in clinical practice. ${ }^{9}$ Future studies should try to explore the learning curve for acquiring competency in various aspects of POCUS.,10

Our study has several limitations. Only a limited number of participants (41) responded to the survey. Majority of the participants worked in operating room and ICU. Only 15\% of the participants had emergency room as the predominant work place. The scenario in emergency room might have been under represented. All the workshops were conducted within Kathmandu valley. The findings of the study cannot be extrapolated to the acute care settings outside Kathmandu, where resource are far more limited. A larger survey, encompassing the doctors working in acute care setting across the nation, can better reveal the scenario of POCUS use and barriers.

To conclude, majority of the participants found the one-day workshop helpful. Doctors from various specialty, working in acute care setting had participated in the survey. Limited access to ultrasound machine, together with lack of adequate knowledge and skills were perceived as major barriers for effective use of POCUS. 
Acknowledgement

All the authors (trainers of the workshop) would like to thank Nepalese Society of Critical Care Medicine (NSCCM) for endorsing the Acute Care Ultrasound workshop.

\section{References}

1. Moore CL, Copel JA. Point-of-care ultrasonography. N Engl J Med. 2011;364:749-57.

https://www.ncbi.nlm.nih.gov/pubmed/21345104

2. Narasimhan M, Koenig SJ, Mayo PH. A whole-body approach to point of care ultrasound. Chest. 2016;150:772-6.

https://www.ncbi.nlm.nih.gov/pubmed/27568582

3. Mayo PH, Beaulieu Y, Doelken P, Feller-Kopman D, Harrod C, Kaplan A, et al. American College of Chest Physicians/ La Societe de Reanimation de Langue Francaise statement on competence in critical care ultrasonography. Chest. 2009;135:1050-60.

https://www.ncbi.nlm.nih.gov/pubmed/19188546

4. Cholley BP, Mayo PH, Poelaert J, Viellard-Baron A, Vignon P, Alhamid $S$, et al. International expert statement on training standards for critical care ultrasonography. Intensive Care Med. 2011;37:1077-83.

https://www.ncbi.nlm.nih.gov/pubmed/21614639

5. Feng M, McSparron Jl, Kien DT, Stone DJ, Roberts DH, Schwartzstein RM, et al. Transthoracic echocardiography and mortality in sepsis: analysis of the MIMIC-III database. Intensive Care Med. 2018;44:884-92.

https://www.ncbi.nlm.nih.gov/pubmed/29806057
6. Shah S, Bellows BA, Adedipe AA, Totten JE, Backlund BH, Sajed D. Perceived barriers in the use of ultrasound in developing countries. Crit Ultrasound J. 2015;7:28.

https://www.ncbi.nlm.nih.gov/pubmed/26123609

7. Fentress M, Heyne TF, Barron KR, Jayasekera N. Point-of-care ultrasound in resource-limited settings: Common applications. South Med J. 2018;111:424-33.

https://www.ncbi.nlm.nih.gov/pubmed/29978229

8. Mayo P, Arntfield R, Balik M, Kory P, Mathis G, Schmidt G, et al. The ICU research agenda on critical care ultrasonography. Intensive Care Med. 2017;43:1257-69.

https://www.ncbi.nlm.nih.gov/pubmed/28271320

9. Atkinson $\mathrm{P}$, Bowra J, Lambert M, Lamprecht $\mathrm{H}$, Noble V, Jarman B. International federation for emergency medicine point of care ultrasound curriculum. CJEM. 2015;17:161-70.

https://www.ncbi.nlm.nih.gov/pubmed/26052968

10. Shrestha GS, Upadhyay B, Shahi A, Jaya Ram KC, Joshi $P$, Poudyal BS. Sonographic measurement of optic nerve sheath diameter: How steep is the learning curve for a novice operator? Indian J Crit Care Med. 2018;22:646-9.

https://www.ncbi.nlm.nih.gov/pubmed/30294130 